\title{
Optimal BESS Scheduling Strategy in Microgrids Based on Genetic Algorithms
}

\author{
Dorian-Octavian Sidea, Lucian Toma, Mihai Sanduleac, Irina Picioroaga, Valentin-Adrian Boicea \\ Department of Electrical Power Systems \\ University "Politehnica" of Bucharest \\ Bucharest, Romania
}

\begin{abstract}
Microgrids represent a promising concept able to solve problems specific to the traditional power systems, such as reliability and environmental issues, through a multi-sources coordination, including renewable energy integration and storage devices. In order to overcome the challenges of variation in load and generation, microgrids need efficiently operation and control. The paper proposes a scheduling framework for the microgrid operation considering renewable sources generation and battery energy storage system (BESS).
\end{abstract}

Index Terms - battery energy storage system, genetic algorithm, microgrid, renewable energy sources

\section{INTRODUCTION}

The accelerated development of the society and industrial sectors consisting in a substantial increase in energy consumption, has revealed many problems with the classical model of centralized generation, such as environmental impact and low reliability in case of extreme weather and other special events. Under these circumstances, power systems may find themselves incapable to respond to the new levels of energy demand, in terms of power quantity.

Defined as a cluster of loads, distributed generators and energy storage devices aggregated within the boundaries of a certain electrical area [1],[2], the microgrid concept represents a promising Smart Grid solution, able to improve the sustainability, reliability and efficiency of current power systems. Microgrids were initially introduced to facilitate the integration of distributed generation, as an efficient alternative to the centralized generation and bulk transmission [3],[4],[5]. In recent years, the microgrids deployment has seen an increased interest given their capacity to operate either in isolated or grid-connected mode [6]. A microgrid can function independently of the main grid, relying only on local resources, resulting in improved reliability and resilience against major outages [7],[8]. Additionally, this concept provides the flexibility needed to incorporate renewable sources, thus contributing to a reduction in the negative environmental impact of traditional power systems.
As the intermittent and stochastic nature of renewable energy sources brings with it many challenges in terms of control and operation of the microgrid, the energy storage devices represent an effective solution to cope with these variations [9],[10],[11],[12].

For an efficient microgrid operation, accurate load demand and renewable energy generation forecasting models are necessary to be implemented. In [13], a three stages approach based on Bender's decomposition is proposed for sizing and scheduling the energy storage system and microturbine, considering also PV generation. A robust optimization model is presented in [14], where the generation scheduling at the Point of Common Coupling (PCC) is analyzed in a microgrid including both wind and PV generation. Despise their modeling accuracy, conventional optimization techniques show a slow convergence time when applied in multi scenario management studies. Therefore, numerous recent papers address heuristic optimization techniques to solve the optimal day-ahead scheduling problem, due to their lowcomputational complexity and algorithmic modeling flexibility [6],[15],[16],[17],[18].

In this paper, a genetic algorithm with a modified mutation function is developed in order to minimize the active power imbalance in a multi-source microgrid, including storage, wind and PV generation. The paper is organized as follows: Section II describes the analyzed microgrid and formulates the scheduling optimization problem. In Section III numerical simulations are performed for multiple daily scenarios and 1, while Section IV provides the conclusions of the study.

\section{OPTIMIZATION PROBLEM FORMULATION}

In this section, a general description of the analyzed microgrid is provided, followed by the optimal scheduling problem formulation.

\section{A. Description of the Analyzed Microgrid}

The microgrid topology considered in this paper consists of local generating units, including a wind power plant 
(WPP) and a photovoltaic power plant (PVPP), electrical loads and a battery energy storage system (BESS), for the active power unbalance reduction. The general architecture of the analyzed microgrid is schematically depicted in Fig. 1. WPP

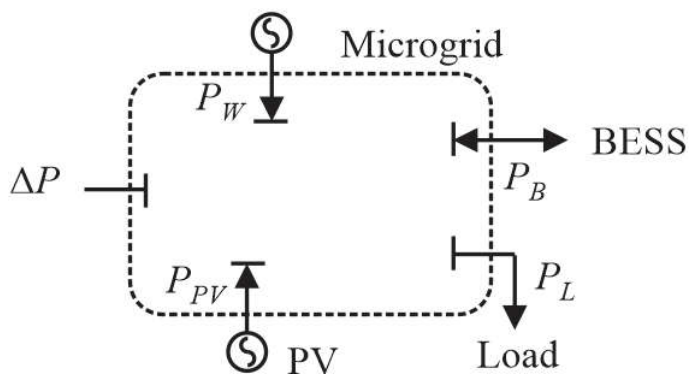

Figure 1. Generic microgrid power exchanges.

\section{B. Scheduling Optimization Model}

The main purpose of the implemented model is to determine the optimal BESS scheduling for the day-ahead, based on the load demand curve and renewable sources (wind and solar) generation forecast, aiming at minimizing the active power unbalance. In this regard, an optimization problem is formulated, having as objective function the sum of the squared active power imbalance for each time interval $k$, within the day-ahead, that is

$$
\min F(x)=\sum_{k=1}^{n}\left[P_{L, k}-P_{W, k}-P_{P V, k}+P_{B, k}\left(x_{k}\right)\right]^{2}
$$

where:

$P_{L} \quad$ is the active power demand [kW];

$P_{W}-$ the active power generated by the WPP [kW];

$P_{P V}$ - the active power generated by the PVPP [kW];

$P_{B}$ - the active power exchanged by the BESS [kW]; positive values stand for consumption, and negative values stand for generation;

$n \quad-$ the number of time intervals considered in the study;

The BESS power at every time interval is expressed as a function of the BESS energy capacity, $W_{\max }[\mathrm{kWh}]$, as

$$
P_{B, k}=x_{x} \cdot W_{\max }
$$

Before applying the optimization algorithm for BESS behavior, the power unbalance for each time interval is calculated based on the forecasted generation and load curves, by:

$$
\Delta P_{k}=P_{L, k}-P_{W, k}-P_{P V, k}
$$

Since the BESS exchanged power can be either positive or negative, in order to avoid opposite operating state, the following restrictions as defined:

- when there is power generation excess $\left(\triangle P_{k}<0\right)$, the BESS can only be charged; therefore, the BESS generation limit, at the time interval $k$, is formulated as

$$
x_{k}^{\min }=\left\{\begin{array}{l}
0 \text { if } \Delta P_{k}<0 \\
x_{\min } \text { otherwise }
\end{array}\right.
$$

- when there is power generation deficit $\left(\Delta P_{k}>0\right)$, the BESS can only be discharged; therefore, the BESS consumption limit, at the time interval $k$, is formulated as follows:

$$
x_{k}^{\max }= \begin{cases}x_{\max } & \text { if } \Delta P_{k}>0 \\ 0 & \text { otherwise }\end{cases}
$$

where $x_{\min }$ and $x_{\max }$ are the actual active power limits, expressed as percentage of the BESS total energy capacity.

By combining the two conditions, the following constraints can be written:

$$
\begin{aligned}
& x_{k}^{\min } \leq x_{k} \leq 0 \text { if } \Delta P_{k}>0 \\
& 0 \leq x_{k} \leq x_{k}^{\max } \text { if } \Delta P_{k}<0
\end{aligned}
$$

For every time interval, the BESS state of charge (SOC) must be maintained within its minimum and maximum limits, $S O C_{\min }$ and $S O C_{\max }$, that is:

$$
S O C_{\min } \leq S O C_{k} \leq S O C_{\max }
$$

In order to determine the state of charge at the end of the $k^{\text {th }}$ time interval $\left(S O C_{k}\right)$, the state of charge variation during this time interval $\left(\triangle S O C_{k}\right)$ is added to the SOC at the end of the previous interval $\left(S O C_{k-1}\right)$, that is:

$$
S O C_{k}=S O C_{k-1}+\triangle S O C_{k}
$$

The numerical simulations begin from a predefined (initial) value denoted by $S O C_{0}$.

The SOC variation during a time interval, $\triangle S O C_{k}$, is determined as follows:

$$
\Delta S O C_{k}=\frac{\Delta W_{k}}{W_{\max }}=\frac{P_{k} \cdot \Delta t}{W_{\max }}=\frac{x_{k} \cdot W_{\max } \cdot \Delta t}{W_{\max }}
$$

Furthermore, considering that the time interval $\Delta t$ is 15 minutes, then $\Delta t=15 / 60=0.25$, (7) becomes:

$$
S O C_{k}=S O C_{k-1}+0.25 \cdot x_{k}
$$

In order to ensure that the state of charge limits are not violated, a penalty function $P_{1}$ is introduced:

$$
P_{1}=\prod_{k=1}^{n} p_{k}
$$

where $p_{k}$ is the penalty coefficient for the time interval $k$ :

$$
p_{k}= \begin{cases}1+S O C_{\min }-S O C_{k} & \text { if } S O C_{k}<S O C_{\min } \\ 1 & \text { if } S O C_{\min } \leq S O C_{k} \leq S O C_{\max } \\ 1+S O C_{k}-S O C_{\max } & \text { if } S O C_{k}>S O C_{\max }\end{cases}
$$

The active power is considered constant during each time interval. Therefore, the energy unbalance is directly proportional with the power unbalance (by a factor of 0.25 ), 
so that the objective function can also be written for energy, instead of power unbalance.

\section{NUMERICAL SIMULATIONS}

\section{A. Simulation algorithm}

The simulations presented in this study are performed under the Matlab 2018 programming environment. The genetic algorithm used for solving the optimization problem presented in section II is based on the default "ga" function form the Matlab Global Optimization Toolbox. A custom mutation function is the most notable customization performed by the authors. Describing the genetic algorithm is out of the scope of this paper, as the purpose is to develop the optimization mathematical model and to study in detail the requirements in terms of BESS capacity in a small grid.

\section{B. Input data}

As mentioned in the mathematical model, only wind and photovoltaic generation units are considered in the simulated microgrid. The following types of data were used for simulations: real values obtained by measurements of PV and WPP units located in distribution networks from Romania, and a generic load curve.

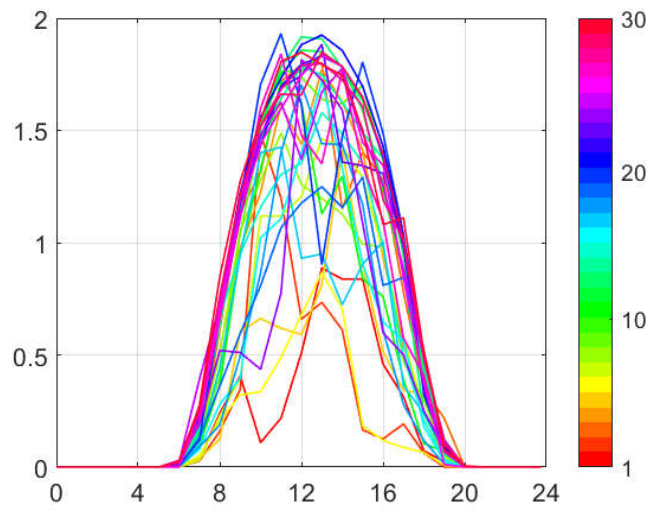

(a)

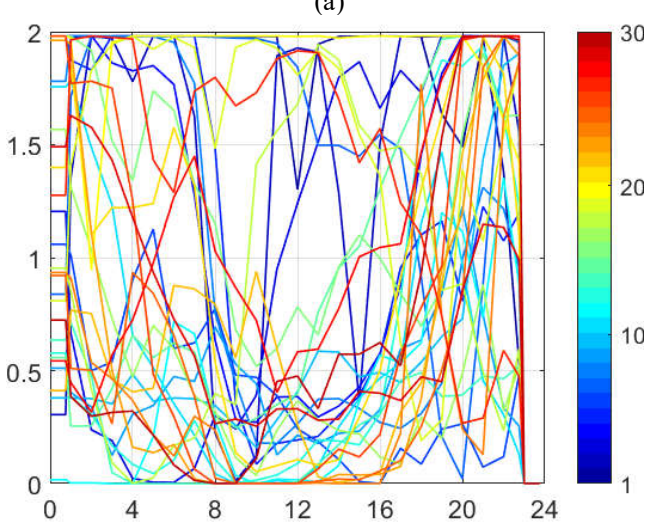

(b)

Figure 2. Daily generated curves active power PVPP (a) and WPP (b) [MW], for every day of April 2018.

The real generated active power data was available for month April 2018 (30 days), with 15-minute granularity. The maximum capacity of both the PVPP and WPP is $2 \mathrm{MW}$. The PVPP generation curves are illustrated in Fig. 2(a), whereas the WPP generation curve is shown in Fig. 2(b). It is easy to see that the PVPP generation is more predictable than the WPP generation. However, summer months in Romania are characterized by higher solar predictability and very low wind speeds, which means that the capacity of the battery should be bigger.

The load curve simulated in this study is a typical load curve, as the one presented in [19]. The load peak-value is $3 \mathrm{MW}$, the lowest value is $1.2 \mathrm{MW}$, whereas the average is 1.8 MW, over a 24-hour time interval presented in Fig. 3. The peak to lower power ratio is 2.5 .

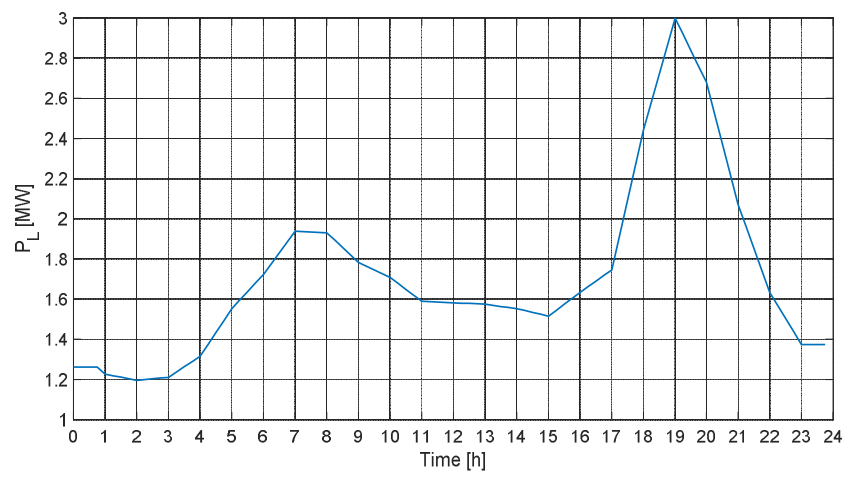

Figure 3. The load profile.

\section{Scenarios}

Multiple scenarios are considered in terms of the power and energy capacity of the BESS in order to identify the best power/energy ratio. Two different power/energy ratios are considered, i.e.: (1) $P_{\max }=80 \% W_{\max }$ and (2) $P_{\max }=25 \% W_{\max }$.

For diversity, a wide range of values for the BESS energy capacity are considered, i.e.:

- when considering both WPP and PVPP, $W_{\max }=2,4,6,8,10$, and $12 \mathrm{MWh}$

- when considering the PVPP only, $W_{\max }=5,10,15,20,25,35$, and $40 \mathrm{MWh}$

The initial charging/discharging operation mode of the BESS is decided by the genetic algorithm.

1) SCENARIO 1: Simulation of the $16^{\text {th }}$ April 2018 curves For better understanding of the requirements on the BESS, the first scenario assumes simulations using the generation curves recorded for one day only.

All simulations assume an initial state of charge $S O C_{0}=$ $50 \%$.

CASE A: The BESS capacity is set to $W_{\max }=8 \mathrm{MWh}$ and $P_{\max }=0.25 \times W_{\max }=2 \mathrm{MW}$. Both WPP and PVPP units are considered.

Figure 4 illustrates the power exchanged by the BESS as bars (left axis), and the SOC as continuous line (right axis).

This particular case reveals that the BESS exchanged active power is limited to the maximum value for almost two 
hours (between 12 and 14), which means that the BESS is not capable of supplying the necessary load within the microgrid.

Correspondingly, the state of charge is limited both at the maximum value for almost three hours (15-18) and at the minimum value for almost another three hours (7-10), showing that the BESS is saturated.

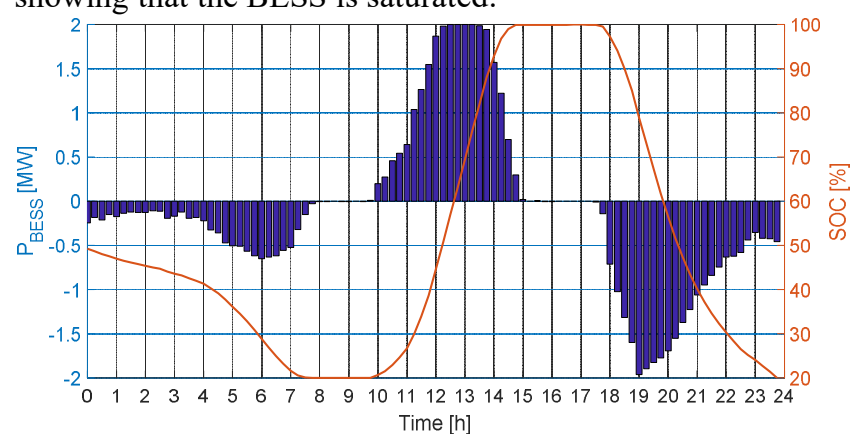

Figure 4. The active power exchanged by the BESS (left axis) and SOC (right axis) for 16-Apr-2018.

In order to overcome the above inconveniencies, multiple BESS energy capacities have been considered. The active power unbalance on every 15-minute time-interval is represented in Fig. 5 for seven energy storage capacity values.

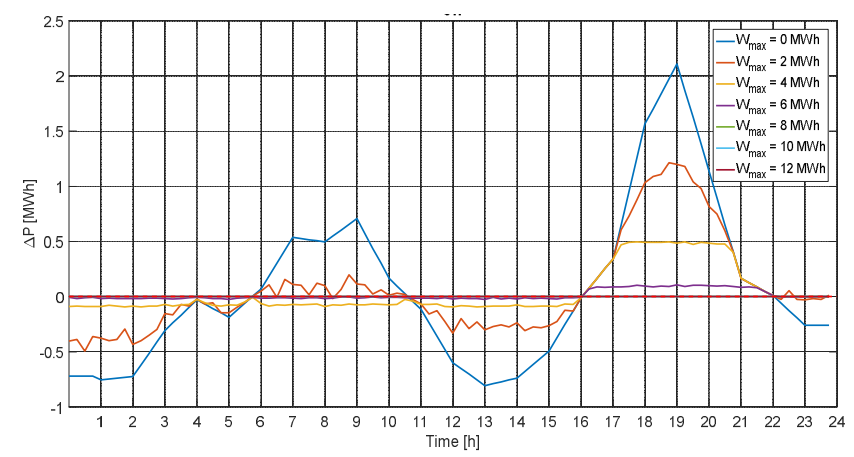

Figure 5. The active power unbalance for various BESS energy capacities, with $25 \%$ power to energy ratio.

Fig. 5 shows that, in the case of a 10 MWh BESS the active power imbalance would be zero for all the 15-minute intervals. For a maximum energy capacity of $6 \mathrm{MWh}$, there is only one time period with a non-zero power imbalance, from 16 to 22 .

The next simulations consider several values for the BESS energy capacity.

Case B: The BESS capacity is set to $W_{\max }=8 \mathrm{MWh}$ and $P_{\max }=0.80 \times W_{\max }$. Both WPP and PVPP units are considered.

Fig. 6 illustrates the active power unbalance resulted during 16-April-2018, also for seven BESS energy capacities. The purpose of increasing the power to energy ratio is to limit the BESS saturation.

For this case, a BESS energy capacity of $W_{\max }=8 \mathrm{MWh}$ is enough to reduce to zero the active power unbalance during the considered day. By comparison, in the previous case (where the power to energy ratio was $25 \%$ ) the necessary energy capacity was $10 \mathrm{MWh}$. Also, for $W_{\max }=6 \mathrm{MWh}$, in both cases only the time period from 16 to 22 is left with a non-zero power unbalance. However, in this case only the energy capacity is too low, while in the previous case both energy and power capacity were too low.

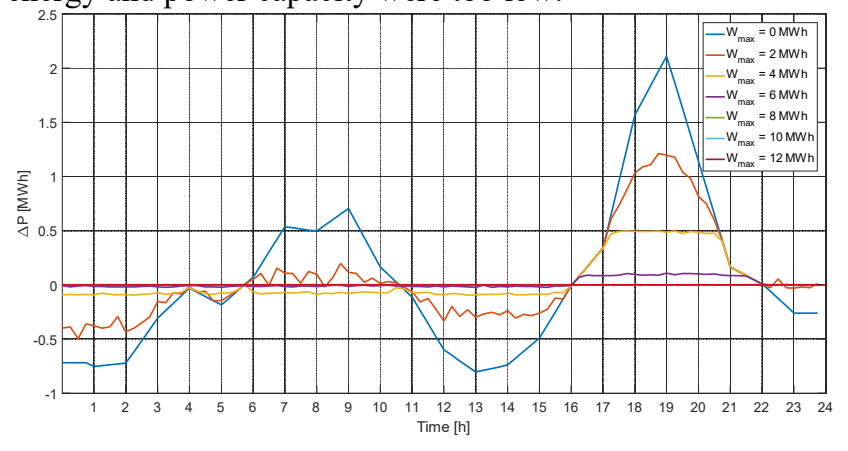

Figure 6. The active power unbalance for various BESS energy capacities, with $80 \%$ power to energy ratio.

Case C: The BESS capacity is set to $W_{\max }=8 \mathrm{MWh}$ and $P_{\max }=0.80 \times W_{\max }$. Only the PVPP unit is considered.

In this case, the PV power plant only is considered as generation source within the microgrid. PVPP generated power is scaled up so that the total active energy generated equals the total active energy demanded by the load. As a result, the maximum power generated by the PVPP increases from $2 \mathrm{MW}$ to $7.35 \mathrm{MW}$, in order cover the 41.2 MWh of total energy demand during the entire day.

The numerical simulations are conducted for nine energy storage capacity values, as shown in Fig. 7.

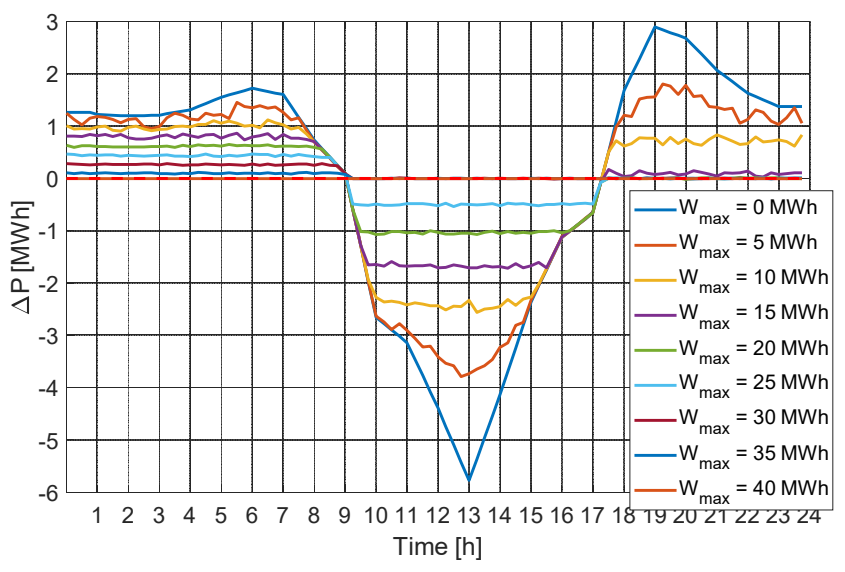

Figure 7. The active power unbalance for various BESS energy capacities, with $80 \%$ power to energy ratio.

Fig 7 shows that a BESS having an energy capacity of 35 $\mathrm{MWh}$ is not enough to eliminate the active power unbalance, because its capacity is not sufficient to store the exceeding energy in the 9:00h-17:00h time interval.

The peak-load is reached at about 19:00h, when the PVPP generates at most $10 \%$ of its installed capacity. Moreover, when the PV generated active power is at its maximum, the 
loads demand is about $1.5 \mathrm{MW} \div 1.7 \mathrm{MW}$, which represents $50 \% \div 57 \%$ of the peak load value of $3 \mathrm{MW}$.

\section{2) SCENARIO 2: Simulation of month April 2018 curves}

In order cover a wider range of generation curves, with a wider diversity, the simulations were expanded for the 30 days of the month April 2018. The BESS characteristics assumed are $W_{\max }=20 \mathrm{MWh}$, and $P_{\max }=80 \% W_{\max }$.

The BESS initial state of charge $\left(S O C_{0}\right)$ represents another important parameter that influences the BESS optimal scheduling. Therefore, three different initial SOC values were considered in this scenario, i.e. $30 \%, 50 \%$, and $80 \%$, respectively.

The daily total positive and negative active energy unbalances $\Delta W^{+}$and $\Delta W^{-}$are calculated as sums of the positive and negative energy imbalances, i.e.:

$$
\Delta W^{+}=\sum_{k \in K_{p}} \Delta P_{k} \cdot \Delta t \text { and } \Delta W^{-}=\sum_{k \in K_{m}} \Delta P_{k} \cdot \Delta t
$$

where

$$
K_{p}=\left\{k \mid \Delta P_{k}>0\right\} \text { and } K_{m}=\left\{k \mid \Delta P_{k}<0\right\}
$$

Then, four $\Delta W^{+}$and $\Delta W^{-}$values are calculated: one for the case without BESS, and three for the considered initial $S O C_{0}$ values, for all the 30 days of month April 2018. The results are presented in Fig. 8 .

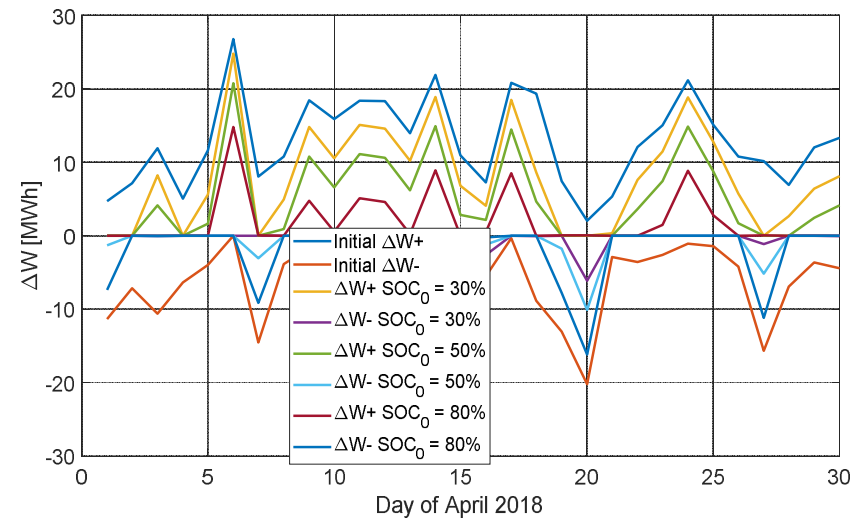

Figure 8. The total positive and negative energy unbalance $\left(\Delta W^{+}\right.$and $\left.\Delta W^{-}\right)$ for the four cases determined for all days of the month April 2018.

Regarding the initial configuration of the microgrid under study, Fig. 8 shows that there are days when the positive energy imbalance is far greater than the negative imbalance (e.g. $6^{\text {th }}, 14^{\text {th }}, 17^{\text {th }}$ or $24^{\text {th }}$ of April) or the opposite (e.g. $20^{\text {th }}$ of April). Also, for some days the values of the $\Delta W^{+}$and $\Delta W^{-}$ are balanced (e.g. $3^{\text {rd }}, 4^{\text {th }}, 6^{\text {th }}$ or $27^{\text {th }}$ of April).

For the cases when there is a significant higher positive energy unbalance, which means that there is an energy deficit within the microgrid, the best initial state of charge value is the highest of the three considered, namely $80 \%$. In the opposite case, when $\Delta W^{+}$is significantly lower than $\Delta W^{-}$, the microgrid operation is characterized by an energy surplus, thus the best $S O C_{0}$ value is the lowest of the considered values, namely $30 \%$.
For the available microgrid data, the minimum BESS energy capacity for which the microgrid energy imbalance can be maintained at zero for the entire day $\left(W_{\min }\right)$ is determined. As shown above, the initial SOC plays an important role in the battery optimal scheduling algorithm, therefore, $W_{\min }$ is determined for each of the three $S O C_{0}$ values considered: $30 \%, 50 \%$ and $80 \%$, respectively. In this manner, for every day of April, three minimum energy capacities are determined, depending on the initial state of charge values: $\Delta W_{\min }^{30}, \Delta W_{\min }^{50}$ and $\Delta W_{\min }^{80}$.

For the optimization problem defined in this paper, if the energy capacity and maximum active power of the BESS are large enough, the optimal solution is obvious. For every time interval the battery should charge/discharge if the active power unbalance is positive/negative, with an amount equal to the absolute value of the active power unbalance.

Therefore, the minimum energy capacity determined for every $S O C_{0}$ value as follows: for every $W$ value starting from $1 \mathrm{MWh}$ (and up to $50 \mathrm{MWh}$, increased by a step of $1 \mathrm{MWh}$ ) the scheduling solution mentioned above is implemented and then it is verified that all the $P_{B}$ and $S O C$ values are within their limits. When a $W_{\min }$ value that satisfied both criteria is found, the values is returned and the algorithm stops. Finally, for every day of April 2018, the minimum energy capacity $W_{\min }$ is determined as the lowest value of the three: $\Delta W_{\min }^{30}$, $\Delta W_{\min }^{50}$ and $\Delta W_{\min }^{80}$. The $S O C_{0}$ value that corresponds to $W_{\min }$ value is denoted as $S O C_{0}^{*}$, which is the best initial state of charge.

Fig. 9 shows the minimum energy capacity and the best initial state of charge resulted from the above described procedure. The $S O C_{0}^{*}$ value, which is the initial state of charge that corresponds to the $W_{\min }$ value, it is indicated within Fig. 9 by the bar fill color as follows: orange $-30 \%$, red $-50 \%$ and blue $-80 \%$.

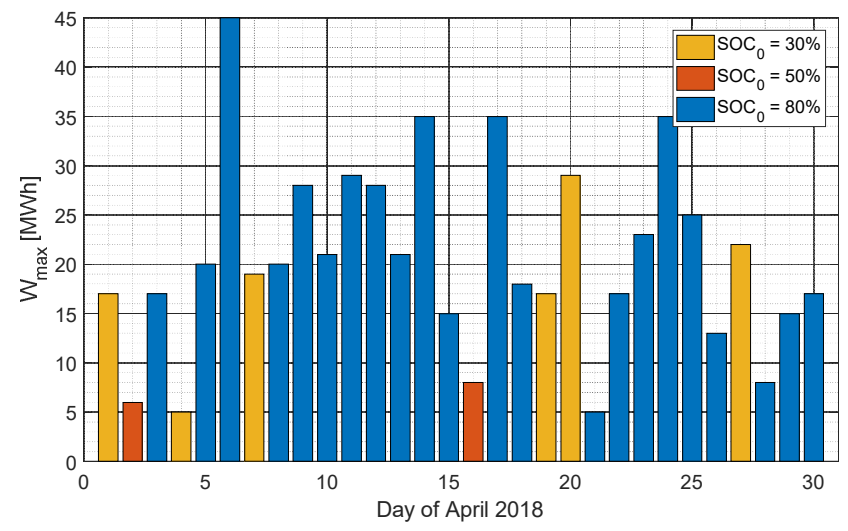

Figure 9. The required BESS energy capacity in order to eliminate the unbalance for every day of April 2018 and the corresponding initial SOC represented by color (orange $-30 \%$, red $-50 \%$ and blue $80 \%$ ).

Figure 10 shows the number of days within which the energy capacity value is able to balance.

From Figs. 9 and 10 it can be seen that a 45 MWh energy capacity BESS is necessary in order to eliminate the 
microgrid active power unbalance for every 15-minute time interval of April 2018. Also, $35 \mathrm{MWh}$ capacity BESS balances 29 days, 29 MWh are enough for 26 days, whereas an $18 \mathrm{MWh}$ capacity can cover 15 days of April.

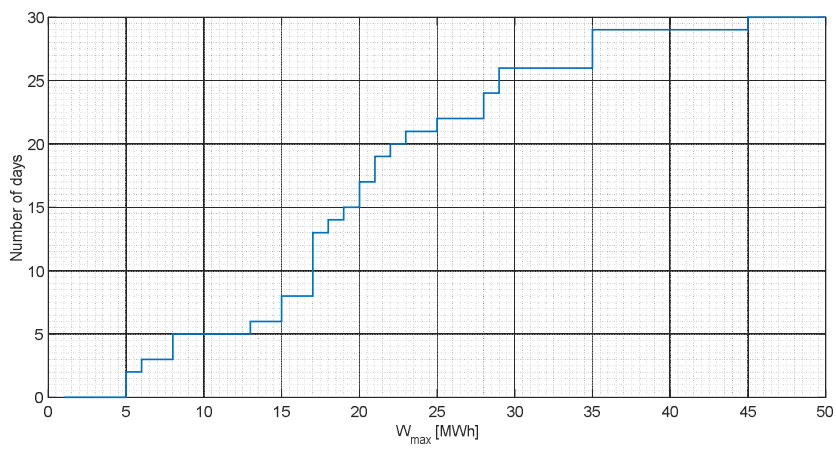

Figure 10. The total number of days from April 2018 that can be balanced by every considered BESS energy capacity value.

With an average power of $1.8 \mathrm{MW}$ for the selected load shape, the daily energy is around $43 \mathrm{MWh}$, which is similar to the maximum BESS capacity required to cover each of the 30 days considered in the analysis. If the more economic 35 MWh capacity is chosen, there is a limited curtailment need of up to $7 \mathrm{MWh}$ for only one day, which is only $7 /(43 \times 30)=$ $0.54 \%$ of the monthly energy, quite acceptable for a reduction of $18 \%$ in installed capacity. The amount of $35 \mathrm{MWh}$ provide $35 / 43 \times 24=19.5$ hours or required energy.

In a previuos work [20], it is shown that a continuous week of operation of the total load in the Romanian power system, while having only renewables resources and the existing nuclear plant, requires a storage capacity capable of supplying energy up to 10 hours. A microgrid is however much more challenging, as it relies only on renewables (no baseline power such as with nuclear energy generation), and requires a much higher $\mathrm{K}$ factor (2.5 as compared to 1.2-1.3 valid at the national level).

\section{CONCLUSIONS}

In conclusion, from the two different power/energy ratios studied, the $80 \%$ version represents a more appropriate solution than the $25 \%$ one, because in high power unbalance situations the latter is not able to provide enough active power, unless the energy capacity is further increased.

In order to ensure a perfect coverage of the daily energy unbalance, an oversizing of the BESS energy capacity is necessary. A cost-effective BESS solution of $35 \mathrm{MWh}$ can be used for a limited curtailment of $0.6 \%$ from monthly energy.

The scenario with PVPP only is characterized by huge necessary BESS energy capacities, especially due to the time difference between the time when the energy is available from the sun (at noon) and when it is used by the loads (in the evening). The $35 \mathrm{MWh}$ BESS solution is equivalent to keeping the microgrid 19.5 hours on storage, which can be expected for a $100 \%$ RES generation in a small microgrid with high load variation. The storage capacity suggests that a BESS solution needs to be combined on medium to long term with complementary solutions such as power to gas (P2G) storage, to address inter season energy resource contribution.

\section{REFERENCES}

[1] I. S. Bae and J. O. Kim, "Reliability Evaluation of Customers in a Microgrid," IEEE Transactions on Power Systems, vol. 23, no. 3, pp. 1416-1422, 2008.

[2] L. Toma, M. Eremia, D. Bica, "Economic operation of distributed energy resources in a microgrid", Proceedings of 2013 IEEE Grenoble PowerTech, Grenoble, France, 16-20 June 2013.

[3] A. G. Tsikalakis and N. D. Hatziargyriou, "Centralized Control for Optimizing Microgrids Operation," IEEE Transactions on Energy Conversion, vol. 23, no. 1, pp. 241-248, 2008.

[4] M. Shahidehpour and J. F. Clair, "A Functional Microgrid for Enhancing Reliability, Sustainability, and Energy Efficiency," The Electricity Journal, vol. 25, no. 8, pp. 21-28, 2012.

[5] A. T. Eseye, D. Zheng, J. Zhang and D. Wei, "Optimal energy management strategy for an isolated industrial microgrid using a Modified Particle Swarm Optimization," in IEEE International Conference on Power and Renewable Energy (ICPRE), Shanghai, China, 2016.

[6] A. Khodaei, "Resiliency-oriented microgrid optimal scheduling," IEEE Transactions on Smart Grid, vol. 5, no. 4, pp. 1584-1591, 2014.

[7] M. H. Amirioun, F. Aminifar and H. Lesani, "Towards Proactive Scheduling of Microgrids Against Extreme Floods," IEEE Transactions on Smart Grid, vol. 9, no. 4, pp. 3900-3902, 2018.

[8] G. Xu, C. Shang, S. Fan, X. Hu and H. Cheng, "A Hierarchical Energy Scheduling Framework of Microgrids With Hybrid Energy Storage Systems," IEEE Access, vol. 6, pp. 2472-2483, 2017.

[9] A. Nagarajan and R. Ayyanar, "Design and scheduling of microgrids using benders decomposition," in IEEE 43rd Photovoltaic Specialists Conference (PVSC), Portland, OR, USA, 2016.

[10] M. Sanduleac, J. Martins, I. Ciornei, M. Albu, L. Toma, F. Pires, L. Hadjidemetriou, R. Saub, "Resilient and Immune by Design Microgrids using Solid State Transformers", Energies 2018, 11(12), 3377.

[11] L. Toma, I. Triştiu, C. Bulac, A.-G. Neagoe-Stefana, "Optimal generation scheduling strategy in a microgrid", IEEE Transportation Electrification Conference and Expo Asia-Pacific (ITEC-BUSAN 2016), Busan, South Korea, 1-4 June 2016.

[12] A. Mazza, E. Bompard, G. Chicco, G., "Applications of power to gas technologies in emerging electrical systems", Renewable and Sustainable Energy Reviews, vol. 92, pp. 794-806

[13] N. Gupta, "Generation scheduling at PCC in grid connected microgrid," in International Conference on Recent Advances and Innovations in Engineering (ICRAIE), Jaipur, India, 2014.

[14] H. Farzin, M. Fotuhi-Firuzabad and M. Moeini-Aghtaie, "A Stochastic Multi-Objective Framework for Optimal Scheduling of Energy Storage Systems in Microgrids," IEEE Transactions on Smart Grid, vol. 8, no. 1, pp. 117-127, 2016.

[15] N. Kumar Paliwal, N. Kumar Singh and A. Kumar Singh, "Energy scheduling of Wind-Battery-Hydro based hybrid microgrid system using heuristic techniques," in IEEE Annual India Conference (INDICON), Bangalore, India, 2016.

[16] W. Bai, I. Eke and K. Y. Lee, "Optimal scheduling of distributed energy resources by modern heuristic optimization technique," in 19th International Conference on Intelligent System Application to Power Systems (ISAP), San Antonio, TX, USA, 2017.

[17] M. De, G. Das, S. Mandal and K. K. Mandal, "A reliable energy management and generation scheduling model in microgrids using modified cuckoo search algorithm," in Emerging Trends in Electronic Devices and Computational Techniques (EDCT), Kolkata, India, 2018.

[18] A. Khodaei, "Provisional Microgrid Planning," IEEE Transactions on Smart Grid, vol. 8, no. 3, pp. 1096-1104, 2017.

[19] E. Bobric, G. Cartina, G. Gheorghe, "Clustering Techniques in Load Profile Analysis for Distribution Stations", Advances in Electrical and Computer Engineering, Vol. 9, No. 1, February 2009

[20] M. Sanduleac, "Energy Storage Needs for Clean Power Systems and the Potential Support from Electrical Vehicles: A Simplified Assessment on Romanian Case", 10th International Conference and Exposition on Electrical and Power Engineering (EPE 2018), Iasi, Romania, 18-19 October 2018. 